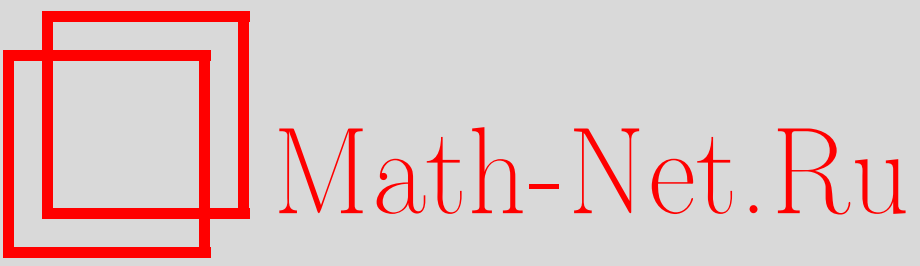

А. В. Аргучинцев, В. П. Поплевко, Задача оптимального управления гиперболической системой с запаздыванием на границе в классе гладких управляющих воздействий, Итоги науки и техн. Сер. Соврем. мат. и ее прил. Темат. обз., 2020, том 183, 14-21

DOI: https://doi.org/10.36535/0233-6723-2020-183-14-21

Использование Общероссийского математического портала Math-Net.Ru подразумевает, что вы прочитали и согласны с пользовательским соглашением

http://www.mathnet.ru/rus/agreement

Параметры загрузки:

IP : 3.85 .73 .92

26 апреля 2023 г., 16:34:57 


\title{
ЗАДАЧА ОПТИМАЛЬНОГО УПРАВЛЕНИЯ ГИПЕРБОЛИЧЕСКОЙ СИСТЕМОЙ С ЗАПАЗДЫВАНИЕМ НА ГРАНИЦЕ В КЛАССЕ ГЛАДКИХ УПРАВЛЯЮЩИХ ВОЗДЕЙСТВИЙ
}

\author{
() 2020 г. $\quad$ А. В. АРГУЧИНЦЕВ, В. П. ПОПЛЕВКО
}

\begin{abstract}
АннотАция. В статье исследуется задача оптимального управления гиперболической системой с дифференциальными связями на границе с учетом запаздывания. Управляющие воздействия выбираются из класса гладких функций, удовлетворяющих поточечным ограничениям. Задачи такого рода возникают, в частности, при моделировании процессов динамики популяций. Предложенный подход основан на использовании «внутренней вариации» управления, сохраняющей гладкость управляющей функции и обеспечивающей выполнение поточечных ограничений. Получена оценка приращения состояния, доказано необходимое условие оптимальности и разработана схема итерационного метода.
\end{abstract}

Ключевые слова: гиперболическая система, система с запаздыванием, необходимое условие оптимальности, гладкое управление.

\section{OPTIMAL CONTROL PROBLEM FOR A HYPERBOLIC SYSTEM WITH DELAY ON THE BOUNDARY IN THE CLASS OF SMOOTH CONTROL ACTIONS}

\author{
(c) 2020 A. V. ARGUCHINTSEV, V. P. POPLEVKO
}

\begin{abstract}
In this paper, we examine the optimal control problem for a hyperbolic system with differential constraints on the boundary, taking into account the delay. Controls are selected from the class of smooth functions that satisfy pointwise constraints. Problems of this type arise, in particular, in modeling the processes of population dynamics. The approach proposed in this paper is based on the use of "internal variations" of the control, which preserves the smoothness of the control function and ensures the fulfillment of pointwise constraints. We obtain an estimate of the state increment, prove a necessary optimality condition, and develop a scheme of an iterative method.
\end{abstract}

Keywords and phrases: hyperbolic system, system with delay, necessary optimality condition, smooth control.

AMS Subject Classification: 49J20, 49M05

1. Постановка задачи. Рассмотрим начально-краевую задачу

$$
\begin{gathered}
x_{t}+x_{s}=f(x, s, t), \\
(s, t) \in \Pi, \quad \Pi=S \times T, \quad S=\left[s_{0}, s_{1}\right], \quad T=\left[t_{0}, t_{1}\right], \\
x\left(s_{0}, t\right)=q(t), \quad t \in[-h, 0], \quad h>0 ;, \quad x\left(s, t_{0}\right)=\mu(s), \quad \mu\left(s_{0}\right)=q\left(t_{0}\right),
\end{gathered}
$$

Работа выполнена при поддержке Российского фонда фундаментальных исследований (проект № 20-07-00407) и совместного проекта РФФИ и Правительства Иркутской области № 20-41-385002. 
где $x=x(s, t)-n$-мерная вектор-функция, $h-$ постоянное запаздывание.

Условия на левом конце определяются из управляемой системы обыкновенных дифференциальных уравнений

$$
x_{t}\left(s_{0}, t\right)=g\left(x\left(s_{0}, t\right), x\left(s_{0}, t-h\right), u(t), t\right), \quad t \in T .
$$

В качестве множества допустимых управлений выберем совокупность гладких на $T r$-мерных вектор-функций $u=u(t)$, удовлетворяющих ограничениям

$$
u(t) \in U, \quad t \in T,
$$

где $U$ - компактное множество.

Требуется найти допустимое управление, доставляющее минимум целевому функционалу

$$
J(u)=\int_{S} \varphi\left(x\left(s, t_{1}\right), s\right) d s .
$$

Введем обозначение $y(t)=x\left(s_{0}, t-h\right)$.

Задача (1)-(4) рассматривается при следующих предположениях:

(i) функции $q(t)$ и $\mu(s)$ непрерывны на $T$ и $S$ соответственно;

(ii) функции $f(x, s, t)$ и $\varphi(x, s)$ непрерывны по совокупности своих аргументов и имеют непрерывные и ограниченные частные производные по $x$;

(iii) функция $g(x, y, u, t)$ непрерывна, непрерывно дифференцируема по своим аргументам и имеет ограниченные производные по $x, y$ и $u$.

Для любого допустимого управления существует единственное обобщенное решение начальнокраевой задачи (1)-(2) из класса непрерывных в П функций, каждая компонента решения $x_{i}, i=$ $1,2, \ldots, n$, непрерывно дифференцируема вдоль любой характеристики системы гиперболических уравнений (см. [1]).

2. Формула приращения функционала. Рассмотрим формулу приращения функционала на двух допустимых процессах: исходном $\{u, x\}$ и проварьированном $\{\widetilde{u}=u+\Delta u, \widetilde{x}=x+\Delta x\}$. Обозначим через $D x=x_{t}+x_{s}$. Здесь $D x=\left(D_{1} x_{1}, \ldots, D_{n} x_{n}\right)$ - обобщенная производная по $x$, каждая компонента которой $D_{i} x_{i}$ непрерывна вдоль соответствующего $i$-го семейства характеристик. Тогда задача в приращениях имеет вид

$$
\begin{gathered}
D \Delta x=\Delta f(x, s, t), \\
\Delta x\left(s_{0}, t\right)=0, \quad t \in[-h, 0], \quad \Delta x\left(s, t_{0}\right)=0, \\
\Delta x_{t}\left(s_{0}, t\right)=\Delta g\left(x\left(s_{0}, t\right), y(t), u(t), t\right) ;
\end{gathered}
$$

здесь

$$
\begin{aligned}
\Delta g\left(x\left(s_{0}, t\right), y(t), u(t), t\right)=g(\widetilde{x}, \widetilde{y}, \widetilde{u}, t)-g & (x, y, u, t)= \\
& =\Delta_{\widetilde{u}} g(x, y, u, t)+\Delta_{\widetilde{x}} g(x, y, \widetilde{u}, t)+\Delta_{\widetilde{y}} g(\widetilde{x}, y, \widetilde{u}, t) .
\end{aligned}
$$

Приращение функционала $\Delta J(u)=J(\widetilde{u})-J(u)$ вычисляется по правилу

$$
\Delta J(u)=\int_{S} \Delta \varphi\left(x\left(s, t_{1}\right), s\right) d s .
$$

Добавим нулевые слагаемые

$$
\iint_{\Pi}\langle\psi(s, t), D \Delta x-f(x, s, t)\rangle d s d t, \quad \int_{T}\left\langle p(t), \Delta x_{t}\left(s_{0}, t\right)-\Delta g\left(x\left(s_{0}, t\right), y(t), u(t), t\right)\right\rangle d t .
$$

Здесь угловыми скобками обозначено скалярное произведение в соответствующем конечномерном евклидовом пространстве. Применяя формулу интегрирования по частям, получим 


$$
\begin{aligned}
\Delta J(u)= & \int_{S} \Delta \varphi\left(x\left(s, t_{1}\right), s\right) d s+\int_{S}\left[\left\langle\psi\left(s, t_{1}\right), \Delta x\left(s, t_{1}\right)\right\rangle-\left\langle\psi\left(s, t_{0}\right), \Delta x\left(s, t_{0}\right)\right\rangle\right] d s- \\
- & -\iint_{\Pi}\left\langle\psi_{t}, \Delta x(s, t)\right\rangle d s d t+\int_{T}\left[\left\langle\psi\left(s_{1}, t\right), \Delta x\left(s_{1}, t\right)\right\rangle-\left\langle\psi\left(s_{0}, t\right), \Delta x\left(s_{0}, t\right)\right\rangle\right] d t- \\
& -\iint_{\Pi}\left\langle\psi_{s}, \Delta x(s, t)\right\rangle d s d t+\left\langle p\left(t_{1}\right), \Delta x\left(s_{0}, t_{1}\right)\right\rangle-\left\langle p\left(t_{0}\right), \Delta x\left(s_{0}, t_{0}\right)\right\rangle- \\
& -\int_{T}\left\langle p_{t}, \Delta x\left(s_{0}, t\right)\right\rangle d t-\iint_{\Pi}\langle\psi(s, t), \Delta f(x, s, t)\rangle d s d t-\int_{T}\langle p(t), \Delta g(x, y, u, s, t)\rangle d t .
\end{aligned}
$$

Введем вспомогательные функции

$$
\begin{gathered}
H(\psi(s, t), x(s, t), s, t)=\langle\psi(s, t), f(x, s, t)\rangle, \\
h\left(p(t), x\left(s_{0}, t\right), y(t), u(t), t\right)=\left\langle p(t), g\left(x\left(s_{0}, t\right), y(t), u(t), t\right)\right\rangle .
\end{gathered}
$$

Тогда

$$
\Delta h(p, x, y, u, t)=\Delta_{\widetilde{u}} h(p, x, y, u, t)+\Delta_{\widetilde{x}} h(p, x, y, \widetilde{u}, t)+\Delta_{\widetilde{y}} h(p, \widetilde{x}, y, \widetilde{u}, t) .
$$

Приращения $\Delta \varphi, \Delta H, \Delta_{\widetilde{x}} h, \Delta_{\widetilde{y}} h$ разложим по формуле Тейлора первого порядка:

$$
\begin{gathered}
\Delta \varphi\left(x\left(s, t_{1}\right), s\right)=\left\langle\frac{\partial \varphi\left(x\left(s, t_{1}\right), s\right)}{\partial x}, \Delta x\left(s, t_{1}\right)\right\rangle+o_{\varphi}\left(\left|\Delta x\left(s, t_{1}\right)\right|\right), \\
\Delta H(\psi, x, s, t)=\left\langle\frac{\partial H(\psi, x, s, t)}{\partial x}, \Delta x(s, t)\right\rangle+o_{H}(|\Delta x(s, t)|), \\
\Delta_{\widetilde{x}} h\left(p, x\left(s_{0}, t\right), y(t), \widetilde{u}, t\right)=\left\langle\frac{\partial h\left(p, x\left(s_{0}, t\right), y(t), \widetilde{u}, t\right)}{\partial x}, \Delta x\left(s_{0}, t\right)\right\rangle+o_{h}\left(\left|\Delta x\left(s_{0}, t\right)\right|\right), \\
\Delta_{\widetilde{y}} h\left(p, \widetilde{x}\left(s_{0}, t\right), y(t), \widetilde{u}, t\right)=\left\langle\frac{\partial h\left(p, \widetilde{x}\left(s_{0}, t\right), y(t), \widetilde{u}, t\right)}{\partial y}, \Delta y(t)\right\rangle+o_{h}(|\Delta y(t)|) .
\end{gathered}
$$

Преобразуем слагаемое

$$
\frac{\partial h\left(p, \widetilde{x}\left(s_{0}, t\right), y(t), \widetilde{u}, t\right)}{\partial y}=\Delta_{\widetilde{u}} \frac{\partial h\left(p, \widetilde{x}\left(s_{0}, t\right), y(t), u, t\right)}{\partial y}+\frac{\partial h\left(p, \widetilde{x}\left(s_{0}, t\right), y(t), u, t\right)}{\partial y},
$$

где

$$
\frac{\partial h\left(p, \widetilde{x}\left(s_{0}, t\right), y(t), u, t\right)}{\partial y}=\Delta_{\widetilde{x}} \frac{\partial h\left(p, x\left(s_{0}, t\right), y(t), u, t\right)}{\partial y}+\frac{\partial h\left(p, x\left(s_{0}, t\right), y(t), u, t\right)}{\partial y} .
$$

Рассмотрим следующее выражение:

$$
\begin{gathered}
\int_{T}\left\langle\frac{\partial h\left(p, x\left(s_{0}, t\right), y(t), u, t\right)}{\partial y}, \Delta y\right\rangle d t= \\
=\int_{T}\left\langle\frac{\partial h\left(p(t), x\left(s_{0}, t\right), x\left(s_{0}, t-h\right), u(t), t\right)}{\partial y}, \Delta x\left(s_{0}, t-h\right)\right\rangle d t= \\
=\left\{\theta=t-h, \theta \in\left[t_{0}-h, t_{1}-h\right]\right\}= \\
=\int_{t_{0}-h}^{t_{0}}\left\langle\frac{\partial h\left(p(\theta+h), x\left(s_{0}, \theta+h\right), x\left(s_{0}, \theta\right), u(\theta+h), \theta+h\right)}{\partial y}, \Delta x\left(s_{0}, \theta\right)\right\rangle d \theta+ \\
+\int_{t_{0}}^{t_{1}-h}\left\langle\frac{\partial h\left(p(\theta+h), x\left(s_{0}, \theta+h\right), x\left(s_{0}, \theta\right), u(\theta+h), \theta+h\right)}{\partial y}, \Delta x\left(s_{0}, \theta\right)\right\rangle d \theta
\end{gathered}
$$


Возвращаясь к переменной $t$, получаем

$$
\int_{t_{0}}^{t_{1}-h}\left\langle\frac{\partial h\left(p(t+h), x\left(s_{0}, t+h\right), x\left(s_{0}, t\right), u(t+h), t+h\right)}{\partial y}, \Delta x\left(s_{0}, t\right)\right\rangle d t .
$$

Потребуем, чтобы функции $\psi(s, t), p(t)$ являлись решениями следующей сопряженной задачи:

$$
\begin{gathered}
\psi_{t}+\psi_{s}=-H_{x}(\psi, x, s, t), \quad \psi\left(s, t_{1}\right)=-\varphi_{x}\left(x\left(s, t_{1}\right), s\right), \quad \psi\left(s_{1}, t\right)=0 ; \\
p_{t}= \begin{cases}-h_{x}[t]-h_{y}[t+h]-\psi\left(s_{0}, t\right), & t \in\left[0 ; t_{1}-h\right], \\
-h_{x}[t]-\psi\left(s_{0}, t\right), & t \in\left[t_{1}-h ; t_{1}\right]\end{cases} \\
p\left(t_{1}\right)=0, \quad p(t) \equiv 0, \quad t>t_{1} .
\end{gathered}
$$

Здесь

$$
h_{x}[t]=h_{x}\left(p(t), x\left(s_{0}, t\right), y(t), u(t), t\right), \quad h_{y}[t+h]=h_{y}\left(p(t+h), x\left(s_{0}, t+h\right), x\left(s_{0}, t\right), u(t+h), t+h\right) .
$$

Тогда формула приращения функционала примет следующий вид:

$$
\Delta J(u)=-\int_{T} \Delta_{\widetilde{u}} h\left(p(t), x\left(s_{0}, t\right), y(t), u(t), t\right) d t+\eta .
$$

Здесь

$$
\begin{aligned}
\eta=\int_{S} o_{\varphi}\left(\left|\Delta x\left(s, t_{1}\right)\right|\right) d s+\iint_{\Pi} o_{H}(|\Delta x(s, t)|) d s d t+ \\
+\int_{T}\left[o_{h}\left(\left|\Delta x\left(s_{0}, t\right)\right|\right)+\left\langle\Delta_{\widetilde{u}} h_{x}\left(p(t), x\left(s_{0}, t\right), y(t), u(t), t\right), \Delta x\left(s_{0}, t\right)\right\rangle\right] d t+ \\
\quad+\int_{T}\left[o_{h}(|\Delta y(t)|)+\left\langle\Delta_{\widetilde{u}} h_{y}\left(p(t), \widetilde{x}\left(s_{0}, t\right), y(t), u(t), t\right), \Delta y(t)\right\rangle\right] d t+ \\
\left.+\int_{T}\left\langle\Delta_{\widetilde{x}} h_{y}\left(p(t), x\left(s_{0}, t\right), y(t), u(t), t\right), \Delta y(t)\right\rangle\right] d t .
\end{aligned}
$$

Покажем (по аналогии с [1]), что при выполнении (5) справедлива оценка

$$
\gamma(t)=\max _{(\xi, \tau) \in \Pi(t)}|\Delta x(\xi, \tau)| \leqslant L_{3} \int_{t_{0}}^{t}|\Delta u(\tau)| d \tau, \quad \Pi(t)=\{(\xi, \tau) \in \Pi: \tau \leqslant t\} .
$$

Для этого рассмотрим интегральный вариант системы (1):

$$
\Delta x_{i}(s, t)=\Delta x_{i}\left(\xi^{(i)}(s, t), \tau^{(i)}(s, t)\right)+\int_{\tau^{(i)}}^{t} \Delta f_{i}(x(\xi, \tau), \xi, \tau) d \tau
$$

где $\left(\xi^{(i)}(s, t), \tau^{(i)}(s, t)\right)$ - начальная точка характеристики $\xi=s^{(i)}(s, t, \tau)$. Введем обозначение

$$
\gamma^{0}(t)=\max _{t_{0} \leqslant \tau \leqslant t}\left|\Delta x\left(s_{0}, \tau\right)\right| .
$$

По предположению задачи

$$
\begin{gathered}
|\Delta f(x, s, t)|=|f(\widetilde{x}, s, t)-f(x, s, t)| \leqslant L|\widetilde{x}-x|, \\
|\Delta g(x, y, u, t)|=|g(\widetilde{x}, \widetilde{y}, \widetilde{u}, t)-g(x, y, u, t)| \leqslant L_{1}(|\widetilde{x}-x|+|\widetilde{y}-y|+|\widetilde{u}-u|) ;
\end{gathered}
$$


здесь $L$ - константа Липшица для функции $f, L_{1}$ - константа Липшица для функции $g$. С учетом (10),(11) имеем оценку

$$
\gamma(t) \leqslant \gamma^{0}(t)+L \int_{t_{0}}^{t} \gamma(\tau) d \tau
$$

Далее, из (5) следует, что

$$
\left|\Delta x\left(s_{0}, t\right)\right| \leqslant \int_{t_{0}}^{t}|\Delta g(x, y, u, \tau)| d \tau \leqslant L_{1} \int_{t_{0}}^{t}\left(\left|\widetilde{x}\left(s_{0}, \tau\right)-x\left(s_{0}, \tau\right)\right|+|\widetilde{y}(\tau)-y(\tau)|+|\widetilde{u}(\tau)-u(\tau)|\right) d \tau .
$$

Имеем

$$
\begin{aligned}
\int_{t_{0}}^{t}|\Delta y(\tau)| d \tau & =\int_{t_{0}}^{t}|\widetilde{y}(\tau)-y(\tau)| d \tau= \\
& =\int_{t_{0}}^{t}\left|\widetilde{x}\left(s_{0}, \tau-h\right)-x\left(s_{0}, \tau-h\right)\right| d \tau=\left\{\tau-h=\theta, \theta \in\left[t_{0}-h, t_{1}-h\right]\right\}= \\
& =\int_{t_{0}-h}^{t_{0}}\left|\widetilde{x}\left(s_{0}, \theta\right)-x\left(s_{0}, \theta\right)\right| d \theta+\int_{t_{0}}^{t}\left|\widetilde{x}\left(s_{0}, \theta\right)-x\left(s_{0}, \theta\right)\right| d \theta=\int_{t_{0}}^{t}\left|\widetilde{x}\left(s_{0}, \tau\right)-x\left(s_{0}, \tau\right)\right| d \tau
\end{aligned}
$$

Тогда

$$
\left|\Delta x\left(s_{0}, t\right)\right| \leqslant 2 L_{1} \int_{t_{0}}^{t}\left|\Delta x\left(s_{0}, \tau\right)\right| d \tau+L_{1} \int_{t_{0}}^{t}|\Delta u(\tau)| d \tau
$$

Из этого неравенства получим

$$
\gamma^{0}(t) \leqslant 2 L_{1} \int_{t_{0}}^{t} \gamma^{0}(\tau) d \tau+L_{1} \int_{t_{0}}^{t}|\Delta u(\tau)| d \tau
$$

Отсюда по лемме Гронуолла-Беллмана имеем

$$
\gamma^{0}(t) \leqslant L_{2} \int_{t_{0}}^{t}|\Delta u(\tau)| d \tau, \quad L_{2}=L_{1} \cdot e^{2 L_{1}\left(t_{1}-t_{0}\right)}
$$

Подставим это неравенство в (12) и повторно применим лемму Гронуолла-Беллмана. В результате получим искомую оценку $(9)$, в которой $L_{3}=L_{2} \cdot e^{L\left(t_{1}-t_{0}\right)}$.

В классе вариаций $\Delta u=\widetilde{u}(t)-u(t)$, малых по норме пространства $C(T)$, формулу приращения (8) можно линеаризовать:

$$
\Delta J(u)=-\int_{T}\left\langle h_{u}\left(p(t), x\left(s_{0}, t\right), y(t), u(t), t\right), \Delta u(t)\right\rangle d t+\eta_{1},
$$

где $\eta_{1}=\eta+o\left(\|\Delta u\|_{C(T)}\right)$. При дополнительном предположении выпуклости множества $U$ отсюда вытекает базовое интегральное необходимое условие оптимальности в исходной задаче оптимального управления.

Теорема 1. Для оптимальности управления и $(t)$ необходимо, чтобы оно являлосъ решением следующей задачи:

$$
I(v)=\int_{T}\left\langle h_{u}\left(p(t), x\left(s_{0}, t\right), y(t), u(t), t\right), v(t)\right\rangle d t \rightarrow \max , \quad v(\cdot) \in C^{1}(T, U) .
$$


Если бы исходная задача оптимального управления исследовалась в классе ограниченных и измеримых управлений, то из (13) сразу бы вытекало условие оптимальности вида дифференциального принципа максимума:

$$
\left\langle h_{u}\left(p(t), x\left(s_{0}, t\right), y(t), u(t), t\right), u(t)\right\rangle=\max \left\langle h_{u}\left(p(t), x\left(s_{0}, t\right), y(t), u(t), t\right), v\right\rangle, \quad v \in U, \quad t \in T .
$$

3. Необходимое условие оптимальности. В силу того, что допустимые управления принадлежат классу гладких функций, используем идею общей методики (см. [1]), основанной на применении неклассических вариаций, обеспечивающих гладкость допустимых управлений. Предлагаемый подход основан на использовании «внутренней вариации» управления, которую применил Л. Е. Забелло в [2,3] для получения необходимых условий оптимальности в задаче оптимального управления системами обыкновенных дифференциальных уравнений с запаздыванием. Автор цитированных работ рассматривал в качестве управляющих воздействий непрерывные кусочно дифференцируемые функции, варьируемые на подмножестве области определения управлений.

В отличие от указанного выше подхода будем строить вариацию на всем отрезке $T$, что гарантирует сохранение гладкости управляющих воздействий. Проварьированное управление конструируется по правилу

$$
u_{\varepsilon, \delta}(t)=u(t+\varepsilon \delta(t)), \quad t \in T
$$

где $\varepsilon \in[0,1]$ - параметр варьирования, $\delta(t)$ - непрерывно дифференцируемая функция, удовлетворяющая условию $t_{0} \leqslant t+\delta(t) \leqslant t_{1}, t \in T$. Так как допустимые управления - гладкие функции, используем следующее разложение:

$$
\Delta u=\dot{u}(t) \varepsilon \delta(t)+o(\varepsilon) .
$$

С помощью оценки (9) получим

$$
\Delta J(u)=-\varepsilon \int_{T}\left\langle h_{u}, \dot{u}\right\rangle \delta(t) d t+o(\varepsilon) .
$$

Отсюда в силу произвольности $\delta(t)$, по аналогии с результатами работы [1], вытекает следующая теорема.

Теорема 2. Если процесс $\{u(t), x(t)\}$ является оптимальным в рассматриваемой задаче, то выполняется условие

$$
\omega(t)=\left\langle h_{u}\left(p(t), x\left(s_{0}, t\right), y(t), u(t), t\right), \dot{u}(t)\right\rangle=0, \quad t \in T,
$$

где $p(t)$ - решение сопряженной задачи (6), (7).

4. Метод улучшения гладких управлений. Опишем общую схему метода.

1. Выберем произвольное допустимое управление $u^{0}=u^{0}(t)$ и положим $k=0$.

2. По управлению $u^{k}$ строим решения $x^{k}, \psi^{k}, p^{k}$ прямой и сопряженной задач.

3. На полученных решениях вычисляется значение функционала $J^{k}=J\left(u^{k}\right)$ и строится функция

$$
\omega_{k}(t)=\left\langle h_{u}\left(p^{k}(t), x^{k}\left(s_{0}, t\right), y^{k}(t), u^{k}, t\right), \dot{u}^{k}\right\rangle
$$

Далее, проверяется условие оптимальности $\omega_{k}(t)=0$. Если оно выполнено, то метод заканчивает свою работу.

4. Если данное управление не удовлетворяет условию оптимальности, строится его гладкая вариация, которая имеет вид

$$
u_{\varepsilon_{k}}^{k}(t)=u^{k}\left(t+\varepsilon_{k} \delta_{k}(t)\right), \quad \delta_{k}(t)=\frac{\left(t-t_{0}\right)\left(t_{1}-t\right) \omega_{k}(t)}{\left(t_{1}-t_{0}\right) \max _{t \in T}\left|\omega_{k}(t)\right|} .
$$

Параметр $\varepsilon_{k}$ определяем из условия

$$
\varepsilon_{k}: J\left(u_{\varepsilon_{k}}^{k}\right)=\min J\left(u_{\varepsilon}^{k}\right), \quad \varepsilon \in[0,1]
$$

Случай, когда найденное значение этого параметра близко к нулю, соответствует неулучшению функционала на шаге метода. 
5. В качестве очередного приближения выбирается $u^{k+1}(t)=u_{\varepsilon_{k}}^{k}(t)$ и итерационный процесс продолжается. Критерием остановки служит одна из ситуаций, полученных на $k$-й итерации метода:

(а) выполнение с заданной точностью необходимого условия оптимальности для функции $u^{k}(t)$. Например, близость к нулю функции $\omega_{k}(t)$ в каждой точке $t \in T$ можно гарантировать, если справедливо неравенство $\max _{t \in T}\left|\omega_{k}(t)\right| \leqslant 10^{-5}$;

(b) неулучшение значения функционала, полученного на предыдущей $(k-1)$-й итерации, например, $J^{k}-J^{k-1}>10^{-6}$. Последовательность управлений, генерируемая методом, является релаксационной (см. [1]).

5. Численный эксперимент. В квадрате $S \times T=[0 ; 5] \times[0 ; 5]$ рассмотрим следующую задачу оптимального управления:

$$
\begin{gathered}
x_{t}+x_{s}=x+f(s, t), \\
x_{t}(0, t)=u \cdot x(0, t-1) ; \quad x(0, t)=0,3 \cdot t, \quad t \in[-1 ; 0], \\
x(s, 0)=0, \quad u(t) \in[0,4] .
\end{gathered}
$$

Целевой функционал имеет вид

$$
J(u)=\frac{1}{2} \int_{S}(x(s, 5)-\bar{x}(s))^{2} d s \rightarrow \min , \quad u \in U .
$$

а вспомогательные функции и сопряженная задача -

$$
\begin{gathered}
H(\psi, x, s, t)=\psi \cdot(x+f(s, t)), \\
\psi_{t}+\psi_{s}=-\psi, \quad \psi(s, 5)=\bar{x}(s)-x(s, 5), \quad \psi\left(s_{1}, t\right)=0 ; \\
h(p, x, y, u, t)=p \cdot u \cdot y ;
\end{gathered}
$$

здесь $x(0, t-1)=y(t), p(5)=0$,

$$
p_{t}= \begin{cases}-p \cdot u-\psi(0, t), & t \in[0 ; 4), \\ -\psi(0, t), & t \in[4 ; 5] .\end{cases}
$$

Вычисления проводились при следующем наборе функций: $f(s, t)=0,1 e^{-s} \cos t$, функция $\bar{x}(s)=\bar{x}(s, 5)$ подсчитана на управлении $\bar{u}(t)=2(\sin 2 \pi t+1))$, в качестве начального управления была выбрана функция

$$
u^{0}(t)=2 \cos \frac{t}{16}-\sin \frac{t}{2}
$$

Значение функционала равно $J\left(u^{0}\right)=11,5766$. Решение задачи выполнялось описанным выше методом. В следующей таблице представлены результаты расчетов:

\begin{tabular}{|c|c|c|c|}
\hline$t$ & $\bar{u}(t)$ & $u^{0}(t)$ & $u^{k}(t)$ \\
\hline 0 & 2 & 2 & 2 \\
0,4 & 2,620 & 1,800 & 2,431 \\
1 & 1,993 & 1,516 & 2,004 \\
1,5 & 2,009 & 1,309 & 2,035 \\
2,025 & 2,300 & 1,135 & 2,570 \\
2,9 & 0,809 & 0,974 & 0,675 \\
3,575 & 1,112 & 0,973 & 1,053 \\
4,1 & 3,154 & 1,047 & 3,196 \\
5 & 1,968 & 1,304 & 1,892 \\
\hline
\end{tabular}

Здесь $u^{k}(t)$ - управление, полученное за 82 итерации метода. Причина остановки методанеулучшение по значению функционала $\left(J\left(u^{k}\right)=0,1842\right)$. При этом $\max _{t \in T}\left|\omega_{k}(t)\right|=0,0644$. 
Проведенный численный эксперимент показал, что предложенный метод улучшения гладких управляющих воздействий может эффективно применяться для решения задач оптимального управления начально-краевыми условиями гиперболических систем с запаздыванием.

Изложенный подход может быть модифицирован и применен также для задач оптимального управления параболическими уравнениями с запаздыванием на основе методики, предложенной в [4].

\section{СПИСОК ЛИТЕРАТУРЫ}

1. Аргучинцев A. В. Оптимальное управление гиперболическими системами. - М.: Физматлит, 2007.

2. Забелло Л. E. K теории необходимых условий оптимальности в системах с запаздыванием и производной от управления// Диффер. уравн. - 1989. - 25, № 3. - С. 371-379.

3. Забелло Л. Е. Об условиях оптимальности в нелинейных инерционных управляемых системах с запаздыванием// Диффер. уравн. - 1990. - 26, № 8. - С. 1309-1315.

4. Arguchintsev A. V., Poplevko V. P. An optimal control problem by parabolic equation witn boundary smooth control and an integral constraint// Num. Alg. Control Optim. — 2018. — 8, № 2. - P. 193-202.

Аргучинцев Александр Валерьевич

Иркутский государственный университет

E-mail: arguch@math.isu.ru

Поплевко Василиса Павловна

Иркутский государственный университет

E-mail: vasilisa@math.isu.ru 